\title{
Current therapy of spinal cord injury
}

\author{
Hirotaka Oikawa ${ }^{1}$, Takahiko Fujikawa ${ }^{1,2}$ and Shunji Tomatsu ${ }^{3}$ \\ ${ }^{1}$ Department of Pharmaceutical Sciences, Suzuka University of Medical Science, Japan \\ ${ }^{2}$ Department of Biochemistry and Proteomics, Mie University Graduate School of Medicine, Japan \\ ${ }^{3}$ Nemours/Alfred I. duPont Hospital for Children, Wilmington, DE, USA
}

\begin{abstract}
The neural axons in the spinal cord that have been damaged by spinal cord injury (SCI) are not regenerated with medicine so far. Several Effective SCI therapies have been now proposed experimentally and/or under investigation clinically. Currently, it has been elucidated that neural axons have a regrowth potential and a number of clinical studies such as some stem cells transplantation and direct administration of inhibitors of neural axons regeneration inhibition have been conducted. However, they require an invasive surgical intervention where the patient's burden is extremely significant. Therefore, development of the non-invasive SCI therapy such as an administration of brain transition drugs is required urgently.
\end{abstract}

We will summarize here the advantage and disadvantage of some potential therapies performed as well as future therapy for SCI.

\section{Introduction}

The spinal cord injury (SCI) refers to a condition that the damage to the spinal cord causes neurologic dysfunction. In the case of neurologic dysfunction owing to SCI, its recovery is desperate, and the pathological condition appears as the incompetence of the body. Originally, neural axons can expand even after injury and produce a recovery of the neuronal network [1]. However, the SCI leads to glial scar formation in the peri-injury area. The glial scars physically inhibit neuronal regeneration due to induction of chondroitin sulfate proteoglycan (CSPG) synthesis. Consequently, damaged spinal axons do not regenerate [2]. Other causes have also been reported such as accumulation of keratan sulfate proteoglycan (KSPG) in peri-injury area. Therefore, if neural regeneration and neural axons outgrowth are promoted by transplantation of stem cells or administration of a drugs, the neuronal network can be reconstructed, and it is conceivable that recovery from neurologic dysfunction is possible. However, effective therapeutic strategy for SCI has not yet been established. In clinical practice, spinal cord protective therapy by a bolus intravenous injection of steroid is performed for acute SCI [3]. It remains controversial in clinical effectiveness and still has arguments for and against [4,5]. Additionally, rehabilitation is performed for chronic SCI, and systemic and local hypothermia have also been attempted as SCI management. In recent years, stem cell transplantation and induction of neural axons regeneration have been attempted, and these approaches had a therapeutic effect. However, these approaches require an invasive surgical procedure.

In this review article, we will introduce some recent approaches to treat SCI and future therapy.

\section{Recent approach}

\section{Cell transplantation}

The advantage of cell transplantation is that it is possible to obtain necessary and sufficient amount of cells by culture technique and also to transfect effective gene. In 1999, Dr. McDonald's research group reported that the neural stem cells differentiated from embryonic stem cells (ES cells) were transplanted to SCI rats. The transplanted neural stem cells were differentiated to neurons, astroglial cells, and oligodendrocytes and improved the neuronal function in SCI rats [6]. In addition, it was reported that a transplantation of human ES cells derived neural stem/progenitor cells recovered motor function in SCI model marmoset [7]. It was proved that SCI is not an untreatable disorder by the accumulation of basic research. Therefore, it was considered to be reproducible in humans. In 2010, a clinical trial was planned to transplant oligodendrocytes derived from human ES cells to SCI patients by Geron, an American pharmaceutical company. However, the clinical trial was discontinued before it was finished due to the economic reasons of the company [8]. Also, this treatment strategy uses human ES cells, thus ethical problems always follow. In such circumstances, Dr. Yamanaka's research group reported the induced pluripotent stem (iPS) cells in 2006 [9]. As a result, it became possible to obtain pluripotent stem cells like ES cells without having an ethical problem. Thereafter, treatments by transplantation of neural stem cells derived from iPS cells to CSI mice were reported $[10,11]$. However, in case of using iPS cells, it has problem of possible formation of teratomas and base of nervous system tumor. Furthermore, in the case of SCI, it has time lag problem that iPS cells take a time to reserve the number of cells necessary for transplantation, thus the required iPS cells are not enough for acute SCI.

On the other hand, spinal cord regeneration by autologous transplantation of olfactory ensheathing cells (OEC) has been attempted. OECs are localized in the olfactory mucosa and olfactory bulb of the organ that transmits olfactory information to the brain. The olfactory mucosa contains neural stem cells capable of replacing

Correspondence to: Hirotaka Oikawa, Department of Pharmaceutical Sciences, Suzuka University of Medical Science, Japan, E-mail: oikawah@suzuka-u.ac.jp

Received: January 28, 2017; Accepted: February 20, 2017; Published: February 22,2017 
nerve cells and OECs capable of axonal growth action, and the nerve regeneration actively occurs in the olfactory mucosa. Therefore, it was conceivable that OECs are effective as transplant for the SCI patients. As a result of OECs transplantation, recovery of motor activity was observed significantly in acute CSI patients, and a certain restorative effect was observed in chronic CSI patients $[12,13]$. Besides that, autologous bone marrow stromal cells transplantation trials $[14,15]$, Schwann cells transplantation [16], and macrophage transplantation [17] have been attempted. However, their actions have not reached a dramatic recovery and/or the effect on humans remains unknown.

\section{Induction of neural axons regeneration}

Multiple molecules that inhibit outgrowth of neural axons after injury have been known and their inhibitors are considered valuable for the reconstruction of the neural circuits after the SCI onset. Nogo has a function of releasing Rho that inhibits neural axons outgrowth by binding with Nogo receptor, and thus clinical trial in CSI patients was performed using Nogo receptor antagonist ATI-355. The clinical trial was completed in 2013. However, the report of ATI-355 has not yet been released. On the other hand, Rho inhibitor cethrin clinical trials are ongoing. Results of the phase I/IIa clinical trial confirmed that cethrin was safe and tolerable, and also suggested that cethrin enhanced motor function recovery in acute SCI patients [18]. Semaphorin3A is known as another inhibitor of neural axons outgrowth. The semaphorin $3 \mathrm{~A}$ inhibitory promotes correct neuronal circuit formation by regulating the neuronal axons at the fetal stage. Thus, the studies of semaphorin $3 \mathrm{~A}$ inhibitors also are underway. In case of spinal cord cut rats after T10 laminectomy, some recovery of motor function has been reported by a combination of direct administration of the semaphorin $3 \mathrm{~A}$ inhibitor to the cut surface and specific and appropriate rehabilitations [19]. The enzyme drug products have also been studied. Neural axons outgrowths are physically inhibited by glial scar produced CSPG in spinal cord periinjury. Thus, CSPG regulation is quite important for neuronal disorder. Chondroitinase $\mathrm{ABC}(\mathrm{C}-\mathrm{ABC})$ is one of the catabolic enzymes for CSPG. Then, $\mathrm{C}-\mathrm{ABC}$ was used for preclinical trial. It was reported that sensory neuron and motor neuron are re-connected when C-ABC was administrated in the subarachnoid of SCI mice [20]. Recently not only CSPG but also KSPG regulation have been attempted. Besides this, Hepatocyte growth factor (HGF) is known as a trophic factor, and it attracts attention because HGF also has neurotrophic factor function same level with brain-derived neurotrophic factor for neurons. HGF administration has been studied for the purpose of neural outgrowth, and then preclinical trial was investigated for an intrathecal HGF administration in contusive cervical SCI marmoset. As a result, it was reported that the SCI-induced paralysis was improved in both upper limbs and lower limbs [21]. However, all of those are invasive therapies using a surgery, and the problem is that the burden on SCI patients is extremely large. Therefore, in the future, a noninvasive medical therapy is desired for neurologic dysfunction caused by SCI.

\section{Prospect of new therapy}

The authors have been researching and developing enzyme drugs for rare disease mucopolysaccharidosis type VII (MPS VII) so far. MPS VII is a disease caused by deficiency or abnormality of $\beta$-glucuronidase (GUS) that is a CSPG catabolic enzyme in the lysosome, and its main symptom is dysostosis. Therefore, we developed an enzyme drug formulation with bone targeting system. The bone targeting system was the theory of adding an aspartic acid chain to GUS (GUS-D6) and selectively targeting to the bone substrate $\mathrm{Ca}^{2+}$. Interestingly, in the GUS-D6 replacement therapy for MPS VII model mice, we found that the GUS-D6 reached the brain with about 3 times higher concentration than native GUS [22]. Furthermore, when we continually administrated to MPS VII model mice at 1 i.v / week for 12 weeks, we observed decrease in CSPG accumulation in cerebral cortex and hippocampus [22]. The current therapies for SCI patients are primarily invasive surgical therapy, and the prognosis is bad due to physical disorder. In addition, because the surgery is a heavy burden for the patients, and noninvasive therapy by drugs is desired greatly. In such fact, GUS-D6 that we developed has following advantage. (1) GUS-D6 is lysosomal enzyme. Thus GUS-D6 can expect a high enzyme activity under acidic conditions of inflamed sites. (2) GUS-D6 can reach the brain. (3) GUS-D6 can depredate CSPG in the central nervous system. Therefore, GUS-D6 can be expected as a new therapeutic agent for neurological dysfunction due to SCI. Eventually, it is possible to establish sustained treatment as a pulse intravenous injection therapy from the acute phase of SCI before glial scar formation, and noninvasive treatment without surgery. Then, it can realize a higher recovery rate of neurological dysfunction and contribute to improvement of QOL of SCI patients.

In recent years, chronic progressive accumulation of CSPG has been reported in the major lesion site in model rats of amyotrophic lateral sclerosis [23]. Therefore, the GUS-D6 enzyme therapy has a potential to be widely applied to diseases caused by such CSPG abnormality. We hope that GUS-D6 enzyme therapy will contribute to the treatment of a wide range of intractable central nervous diseases in the future.

\section{References}

1. David S, Aguayo AJ (1981) Axonal elongation into peripheral nervous system "bridges" after central nervous system injury in adult rats. Science 214: 931-933.

2. Galtrey CM, Fawcett JW (2007) The role of chondroitin sulfate proteoglycans in regeneration and plasticity in the central nervous system. Brain Res Rev 54: 1-18.

3. Bracken MB (2012) Steroids for acute spinal cord injury. Cochrane Database Syst Rev 1: CD001046

4. Michael BB, Mary JS, William FC (1990) A randomized, controlled trial of methylprednisolone or naloxone in the treatment of acute spinal-cord injury. Results of the Second National Acute Spinal Cord Injury Study. N Engl J Med 322: 1405-1411.

5. Hurlbert RJ (2000) Methylprednisolone for acute spinal cord injury: an inappropriate standard of care. J Neurosurg 93(1 Suppl): 1-7.

6. McDonald JW, Liu XZ, Qu Y (1999) Transplanted embryonic stem cells survive, differentiate and promote recovery in injured rat spinal cord. Nat Med 5: 1410-1412.

7. Iwanami A, Kaneko S, Nakamura M (2005) Transplantation of human neural stem cells for spinal cord injury in primates. J Neurosci Res 80: 182-190.

8. Scott CT, Magnus D (2014) Wrongful termination: lessons from the Geron clinical trial Stem Cells Transl Med 3: 1398-1401.

9. Takahashi K, Yamanaka S (2006) Induction of pluripotent stem cells from mouse embryonic and adult fibroblast cultures by defined factors. Cell 126: 663-676.

10. Nori S, Okada Y, Yasuda A (2011) Grafted human-iducedpluripotent stem-cell-derived neurospheres promote motor functional recovery after spinal cord injury in mice. Proc Natl Acad Sci U S A 108: 16825-16830.

11. Fujimoto Y, Abematsu M, Falk A (2012) Treatment of a mouse model of spinal cord injury by transplantation of human induced pluripotent stem cell-derived long-term self-renewing neuroepithelial-like stem cells. Stem Cells 30: 1163-1173.

12. Moriwaki T, Iwatsuki K, Mochizuki-Oda N (2014) Presence of trans-synaptic neurons derived from olfactory mucosa transplanted after spinal cord injury. Spine (Phila Pa 1976) 39: 1267-1273.

13. Wanf S, Lu J, Li YA (2016) Autologous olfactory lamina propria transplantation for chronic spinal cord injury: three-year follow-up outcomes from a prospective doubleblinded clinical trial. Cell Transplant 25: 141-157.

14. Saito F, Nakatani T, Iwase M (2012) Administration of cultures autologous bone marrow stromal cells into cerebrospinal fluid in spinal injury patients: a pilot study. Restor Neurol Neurosci 30: 127-136. 
15. Suzuki Y, Ishikawa N, Omae K (2014) Bone marrow-derived monuclear cell transplantation in spinal cord injury patients by lumbar puncture. Restor Neurol Neurosci 32: 473-482.

16. Martin D, Robe P, Franzen R (1996) Effects of schwann cell transplantation in a contusion model of rat spinal cord injury. J Neurosci Res 45: 588-597.

17. Knoller N, Auerbach G, Fulga V (2005) Clinical experience using incubated autologous macrophages as a treatment for complete spinal cord injury: phase 1 study results. $J$ Neurosurg Spine 3: 173-181.

18. Fehlings MG, Theodore N Harrop J (2011) A phase I/IIa clinical trial of a recombinant Rho protein antagonist in acute spinal cord injury. $J$ Neurotrauma 28: 787-796.

19. Zhang L, Kaneko S, Kikuchi K (2014) Rewiring of regenerated axons by combining treadmill training with semaphoren3A inhibition. Mol Brain 7: 14.

20. Imagama S, Sakamoto K, Tauchi R (2011) Keratan sulfate restricts neural plasticity after spinal cord injury. J Neurosci 31: 17091-17102.

21. Kitamura K, Fujiyoshi K, Yamane J (2011) Human Hepatocyte growth factor promotes functional recovery in primates after spinal cord injury. PLoS One 6: e27706.

22. MontañoAM, Oikawa H, Tomatsu S (2008) Acidic amino acid tag enhances response to enzyme replacement in mucopolysaccharidosis type VII mice.Mol Genet Metab 94 178-189.

23. Mizuno H, Warita H, Aoki M, Itoyama Y (2008) Accumulation of chondroitin sulfate proteoglycans in the microenvironment of spinal motor neurons in amyotrophic lateral sclerosis transgenic rats. J Neurosci Res 86: 2512-2523.

Copyright: $(02017$ Oikawa H. This is an open-access article distributed under the terms of the Creative Commons Attribution License, which permits unrestricted use, distribution, and reproduction in any medium, provided the original author and source are credited. 